\title{
A CENTRAL LIMIT THEOREM FOR CERTAIN NONLINEAR STATISTICS IN REPEATED \\ SAMPLING OF A FINITE POPULATION
}

\author{
D. L. Hawkins \\ Chien-Pai Han \\ Mathematics Department \\ University of Texas at Arlington
}

Technical Report \#312 


\title{
A CENTRAL LIMIT THEOREM FOR CERTAIN NONLINEAR STATISTICS IN REPEATED SAMPLING OF A FINITE POPULATION
}

\author{
D. L. Hawkins \\ Chien-Pai Han \\ University of Texas at Arlington
}

\section{Abstract.}

We prove a central limit theorem for the asymptotic joint distribution of non-linear statistics of the form $\sum_{\ell=1}^{N} Z_{N \ell t} I_{t \ell} I_{t+1, \ell}, 1 \leq t \leq r-1$ and linear statistics of the form $\sum_{\ell=1}^{N}$

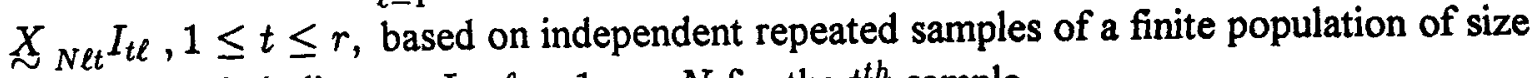
$\mathrm{N}$ with sample indicators $I_{t \ell}, \ell=1, \cdots, N$ for the $t^{\text {th }}$ sample.

Key words and phrases: Capture-recapture, dual-system estimation, asymptotic distribution.

\section{Introduction and Main Results.}

Let $\ell=1, \cdots, N$ index the members of a finite population $S$. Consider sampling $S$ at $r$ consecutive times, or waves, indexed by $t=1, \ldots, r$. The sample for wave $t$ is determined by the random sampling indicators $I_{t \ell}, \ell=1 \cdots, N$, where $I_{t \ell}=1$ if member $\ell$ is in the sample at wave $t$, and $I_{t \ell}=0$ otherwise. Assume that $I_{t \ell}$ and $I_{t^{\prime} \ell^{\prime}}$ are independent if $t \neq t^{\prime}$, and that $\sum_{\ell=1}^{N} I_{t \ell}=n_{t}$ is fixed in advance. Let $f_{t}=n_{t} / N$ denote the sampling fraction, and assume that $E\left(I_{t \ell}\right)=f_{t}$ (e.g. under simple random sampling).

Let $\left\{{\underset{\sim}{X}}_{N \ell t}: 1 \leq \ell \leq N\right\}$ be a triangular array of $m_{x} \times 1$ constant vectors for each $t=1, \cdots, r$, satisfying, as $N \rightarrow \infty$,

$$
\bar{\sim}_{N t} \rightarrow{\underset{\sim}{\mu}}_{t}^{x}, \stackrel{\sim}{\sim}_{N t}^{2} \rightarrow \underset{\sim}{\Gamma}{ }_{t}^{x}
$$

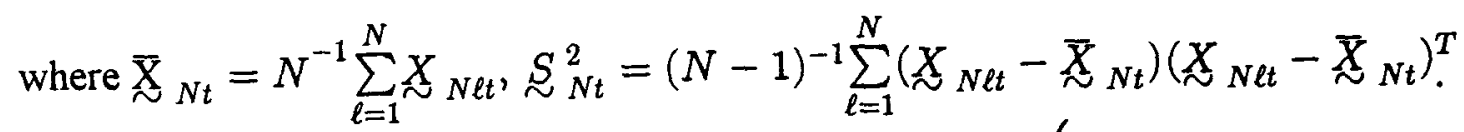

Under the conditions (1.1), a central limit theorem exists (see Hajek (1960), Madow (1948), Rosen (1965), White (1988)) for linear statistics of the form 


$$
L_{N t}=\sum_{\ell=1}^{N} \underset{\sim}{X}{ }_{N \ell t} I_{t \ell}
$$

which are functions of the sample from wave $t$ only.

The purpose of the present paper is to give a central limit theorem for non-linear statistics of the form

$$
\begin{gathered}
{\underset{\sim}{\xi}}_{N t}=\sum_{\ell=1}^{N}{\underset{N}{N \ell t}}_{t \ell} I_{t+1, \ell} I_{t}(1 \leq t \leq r-1 \text { fixed }) \text { and } \\
{\underset{\sim}{\sim}}_{N}=\sum_{t=1}^{r-1} \xi_{N t},
\end{gathered}
$$

where, for $t=1, \cdots, r-1,\left\{{\underset{N}{N \ell t}}_{N}: 1 \leq \ell \leq N\right\}$ is a triangular array of $m_{Z} \times 1$ constant vectors which satisfy (1.1) with limits $\underset{\mathfrak{t}}{Z}$ and $\Gamma_{\mathfrak{\sim}}^{Z}$. Such statistics are common in the so-called dual-system estimation method of sample survey methodology. See Chandrasekaran and Deming (1949), Alho et al (1993), Isaki (1986, 1987), Krotki (1978). Also, such statistics are often used in conjunction with linear statistics of the form (1.2). See Hawkins et al (1995a), where statistics of the form (1.4) were used to define estimates of transition probabilities based on recaptures, which were combined with transition probability estimates from a Markov model based on marginal estimates of the form (1.2).

Since $\underset{\sim}{L_{N t}}$ and ${\underset{\sim}{\sim}}_{N t}$ (or ${\underset{\sim}{\xi}}_{N}$ ) will often occur together in applications, our main result (Theorem 1) gives the asymptotic joint normality of

$$
\left(\underset{\sim}{\stackrel{T}{\sim}}, \cdots, \underset{\sim}{\stackrel{T}{\sim}}{ }_{N r}, \underset{\sim}{\xi_{N 1}^{T}}, \cdots, \underset{\sim}{\xi_{N, r-1}^{T}}\right) .
$$

In this direction we shall assume

(A1) The wave sample sizes $n_{t}, 1 \leq t \leq r$, satisfy $n_{t}=n_{(N)} \cdot \lambda_{t}$, where

$\lambda_{t}>0, \lambda_{1}+\cdots+\lambda_{r}=1$ and $n_{(N)} \rightarrow \infty$, but $n_{(N)} / N \rightarrow 0$, as $N \rightarrow \infty$. (Note that this implies $\left.n_{1}+\cdots+n_{r}=n_{(N)} \cdot\right)$

(A2) $\left(n_{(N)}\right)^{3 / 2} / N \rightarrow \infty$ as $N \rightarrow \infty$.

Also define $g_{N t}=n_{t} n_{t+1} / N$ and $g_{N}=\sum_{t=1}^{r-1} g_{N t}$.

Assumption (A1) is standard for the asymptotic normality of $L_{N t}$ under condition (1.1). Assumption (A2) implies that the expected number of recaptures between samples $t$ and $t+1$, which is $n_{t} n_{t+1} / N$, is $O\left(n_{(N)}^{2} / N\right) \rightarrow \infty$ as $N \rightarrow \infty$. This is a natural assumption for $\xi_{\mathrm{Nt}}$ to be asymptotically normal under condition (1.1). 
Now define the standardized statistics

$$
\begin{aligned}
& \widetilde{L}_{N t}=n_{t}^{-\frac{1}{2}}\left\{L_{N t}-E\left(L_{N t}\right)\right\}, 1 \leq t \leq r, \\
& \underline{\xi}_{N t}=g_{N t}^{-\frac{1}{2}}\left\{\xi_{N t}-E\left(\xi_{N t}\right)\right\}, 1 \leq t \leq r-1, \\
& \widetilde{\xi}_{N}=g_{N}^{-\frac{1}{2}}\left\{\xi_{N}-E\left(\xi_{N}\right)\right\}, \text { and let } \\
& {\underset{\sim}{\widetilde{W}}}_{N}=\left(\widetilde{L}_{N 1}^{T}, \cdots,{\underset{\sim}{\widetilde{L}}}_{N r}^{T},{\underset{\sim}{\tilde{\xi}}}_{N 1}^{T}, \cdots,{\underset{\sim}{\tilde{\xi}}}_{N, r-1}^{T}\right)^{T}
\end{aligned}
$$

Then the main result is

Theorem 1. Under (1.1), (A1) and (A2), $\underset{\sim}{\widetilde{W}} \stackrel{\mathcal{L}}{\rightarrow} N(\underline{0}, \underline{\Sigma})$ as $N \rightarrow \infty$, where $\sum_{\sim}$ is of order $r m_{x}+(r-1) m_{Z}$ and given by

$\sum_{\sim}=\left[\begin{array}{cc}\sum_{\sim} & \stackrel{\sim}{\sim} \\ 0 & \sum_{\sim z}\end{array}\right], \sum_{\sim x}=$ Block diag $\left[\Gamma_{s}^{x}: 1 \leq s \leq r\right]$

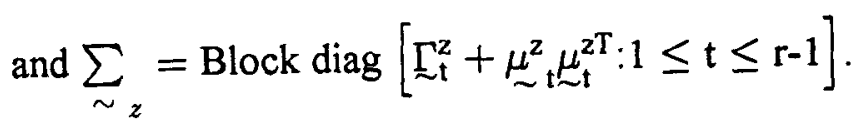

From this result we see that the $L_{N t}$ 's and the ${\underset{\sim}{\prime}}_{N t}^{\prime}$ s are asymptotically (as $N \rightarrow \infty$ ) independent, as are $\xi_{N 1}, \cdots, \xi_{N, r-1}$. However, pre-asymptotically, these statistics are correlated, with covariance of order $\left(n_{(N)} / N\right)^{\frac{1}{2}}$. See the proof of Theorem 1.

Finally, we immediately obtain the asymptotic joint normality of

$$
\widetilde{W}_{N}^{(1)}=\left(\widetilde{L}_{N 1}^{T}, \cdots, \widetilde{L}_{N r}^{T}, \widetilde{\xi}_{N}^{T}\right)^{T}
$$

Corollary 1. Under (1.1), (A1) and (A2), $\underset{\sim}{\underset{\sim}{(1)}} \stackrel{\mathcal{L}}{\rightarrow} N\left(\underset{\sim}{0}, \sum_{\sim}^{(1)}\right)$ where 


$$
\begin{gathered}
\sum_{\sim}^{(1)}=\left[\begin{array}{cc}
\sum_{\sim x} & 0 \\
0 & \sum_{z 1}
\end{array}\right], \\
\sum_{\sim z 1}=\sum_{t=1}^{r-1} \alpha_{t}\left\{\Gamma_{t}^{z}+\mu_{t}^{z} \mu_{t}^{z T}\right\} \text { and } \alpha_{t}=\lambda_{t} \lambda_{t+1} / \sum_{s=1}^{r-1} \lambda_{s} \lambda_{s+1} .
\end{gathered}
$$

\section{Proof of the Central Limit Theorem}

We sketch the proof here, leaving the proofs of the lemmas to the technical report Hawkins and Han (1996).

We use the method of Hajek, which begins with the observation that statistics in the sampling indicators $I_{t \ell}$ have the same distributions as certain rank statistics. Specifically, for each $1 \leq t \leq r$ define i.i.d. $U(0,1)$ variables $V_{N \ell}^{(t)}, \ell=1, \cdots, N$ and let $\underset{\sim}{\sim} \stackrel{(t)}{\sim}=\left(R_{N 1}^{(t)}, \cdots, R_{N N}^{(t)}\right)$ denote the vector of ranks of $V_{N 1}^{(t)}, \cdots, V_{N N}^{(t)}$. Then the random vector $\stackrel{I}{\sim}_{N t}^{*}=\left(I\left(R_{N 1}^{(t)} \leq n_{t}\right), \cdots, I\left(R_{N N}^{(t)} \leq n_{t}\right)\right)$ has the same joint distribution as does $\stackrel{I}{\sim}_{N t}=\left(I_{t 1}, \cdots, I_{t N}\right)$ (conditional on $\left.\sum_{\ell=1}^{N} I_{t \ell}=n_{t}\right)$. Further, since $I_{N 1}, \cdots, I_{N r}$ are assumed independent, if $\left\{V_{N \ell}^{(t)}\right\}$ are independent across $t=1, \cdots, r$, then the vectors $\stackrel{I}{\sim}_{N}^{*}=\left(I_{N 1}^{*}, \cdots, I_{N r}^{*}\right)$ and $I_{N}=\left(I_{N 1}, \cdots, I_{N r}\right)$ have the same distribution (conditional on $\sum_{\ell=1}^{N} I_{t \ell}=n_{t}$ for each $\left.t=1, \cdots, r\right)$, as do $h\left(I_{N}^{*}\right)$ and $h\left(I_{N}\right)$ for any measurable map $h$. The implication of this is that we may assume for distribution theory that the statistics $\underset{\sim}{L}$ and $\underset{\sim}{\sim} N t$ are given with $I_{t \ell}$ replaced by $\beta_{\mathrm{N} \ell \mathrm{t}}=: I\left(R_{N \ell}^{(t)} \leq n_{t}\right), 1 \leq \ell \leq N$. I.e. we can consider instead the statistics

$$
L_{N t}^{o}=\sum_{\ell=1}^{N} \underset{N \ell t}{X} \beta_{N \ell t}
$$




$$
\begin{gathered}
\stackrel{\xi}{\sim}_{N t}^{o}=\sum_{\ell=1}^{N} Z_{N \ell t} \beta_{N \ell t} \beta_{N \ell, t+1}, \\
\xi_{N}^{o}=\sum_{t=1}^{r-1} \stackrel{\xi}{N t}_{N t}^{o}
\end{gathered}
$$

The next step in Hajek's method is to approximate the resulting rank statistics in terms of independent random variables. For linear statistics like ${\underset{\sim}{L}}_{N t}^{o}$ we have Hajek's basic lemma, given here as reformulated by White (1988) and adapted to the present context. Let

$$
\beta_{N \ell t}^{*}=I\left(V_{N \ell}^{(t)} \leq f_{t}\right), 1 \leq \ell \leq N
$$

Note that the $\beta_{N \ell t}^{*}$ are independent over $\ell=1, \cdots, N$.

Lemma 1 . Let $\left\{c_{N \ell t}: 1 \leq \ell \leq N\right\}$ be a triangular array of constant $m \times 1$ vectors for each $t=1, \cdots, r$, satisfying

(C1) $n_{t}^{\frac{1}{2}} \overline{\mathcal{c}}_{N t} \rightarrow 0, n_{t}^{\frac{1}{2}} \mathcal{c}_{N t}^{2} \rightarrow 0$ as $N \rightarrow \infty$ where

$$
\overline{\mathcal{c}}_{N t}=: \frac{1}{N} \sum_{\ell=1}^{N} \underset{\sim}{\sim}{ }_{N \ell t}, \stackrel{c}{\sim}_{N t}^{2}=: \frac{1}{N-1} \sum_{\ell=1}^{N}\left(\mathcal{c}_{N \ell t}-\overline{\mathcal{c}}_{N t}\right)\left(\mathcal{c}_{N \ell t}-\overline{\mathcal{c}}_{N t}\right)^{T}
$$

Let $\bigcup_{N t}=\sum_{\ell=1}^{N} \underset{\sim}{\mathcal{c}}{ }_{N \ell t} \beta_{N \ell t}, \quad \bigcup_{N t}^{*}=\sum_{\ell=1}^{N} \mathcal{\sim}_{N \ell t} \beta_{N \ell t}^{*}$

Then $E\left\|\bigcup_{N t}-U_{N t}^{*}\right\|^{2} \rightarrow 0$ as $N \rightarrow \infty$, each $t=1, \cdots, r$.

The following result extends Lemma 1 to the nonlinear statistics $\xi_{N t}$.

Lemma 2. Let $\left\{c_{N \ell t}: 1 \leq \ell \leq N\right\}$ satisfy (C1), each $t=1, \cdots, r-1$.

Define $\underset{N t}{\epsilon}=\sum_{\ell=1}^{N} \underset{\sim}{\sim}{ }_{N \ell t} \beta_{N \ell t} \beta_{N \ell, t+1}, \stackrel{\epsilon}{\sim}_{N t}^{*}=\sum_{\ell=1}^{N} \underset{N \ell t}{\mathcal{C}} \beta_{N \ell t}^{*} \beta_{N \ell, t+1}^{*}$ 
Then $E\left\|\epsilon_{N t}-\stackrel{\epsilon}{\sim}_{N t}^{*}\right\|^{2} \rightarrow 0$ as $N \rightarrow \infty$, each $t$.

To apply these results to $\widetilde{W}_{N}$ (with $\underset{\sim}{L}{ }_{N t},{\underset{\sim}{\xi}}_{N t}$ replaced by $L_{N t}^{o},{\underset{\sim}{\underbrace{}_{N t}}}^{o}$ ), we first need to decompose the component statistics appropriately. First, since $E\left(L_{N t}^{o}\right)=n_{t} \bar{X}_{N t}$ and $\sum_{\ell=1}^{N} \beta_{N \ell t}=n_{t}$ a.s.,

$$
\hat{\underline{\alpha}}_{N t}=: n_{t}^{-\frac{1}{2}}\left\{L_{N t}^{\mathrm{o}}-E\left(L_{N t}^{\mathrm{o}}\right)\right\}=\sum_{\ell=1}^{N} n_{t}^{-\frac{1}{2}}\left(\mathbb{X}_{N \ell t}-\bar{X}_{N t}\right) \beta_{N \ell t} .
$$

Further, since $E\left(\underline{\xi}_{N t}^{\mathrm{o}}\right)=\frac{1}{N} \sum_{\ell=1}^{N} Z_{N \ell t} g_{N t}=g_{N t} \bar{Z}_{N t}$,

$$
\begin{aligned}
\hat{\delta}_{N t}= & g_{N t}^{-\frac{1}{2}}\left\{\xi_{N t}^{0}-E\left(\xi_{N t}^{0}\right)\right\} \\
= & g_{N t}^{-\frac{1}{2}}\left\{\sum_{\ell=1}^{N} Z_{N \ell t} \beta_{N \ell t} \beta_{N \ell, t+1}-g_{N t} \bar{Z}_{N t}\right\} \\
= & g_{N t}^{-\frac{1}{2}}\left\{\sum_{\ell=1}^{N} Z_{N \ell t} \beta_{N \ell t} \beta_{N \ell, t+1}-n_{t, t+1} \bar{Z}_{N t}\right\} \\
& +g_{N t}^{-\frac{1}{2}}\left\{n_{t, t+1} \bar{Z}_{N t}-g_{N t} \bar{Z}_{N t}\right\} \\
= & g_{N t}^{-\frac{1}{2}} \sum_{\ell=1}^{N}\left(Z_{N \ell t}-\bar{Z}_{N t}\right) \beta_{N \ell t} \beta_{N \ell, t+1}\left(\text { call this }{ }_{1} \widehat{\delta}_{N t}\right), \\
& \left.+g_{N t}^{-\frac{1}{2}} \bar{Z}_{N t} \sum_{\ell=1}^{N}\left\{\beta_{N \ell t} \beta_{N \ell, t+1}-E\left(\beta_{N \ell t} \beta_{N \ell, t+1}\right)\right\} \text { (call this } 2 \stackrel{\hat{\delta}}{\sim}_{N t}\right),
\end{aligned}
$$

where $n_{t, t+1}=: \sum_{\ell=1}^{N} \beta_{N \ell t} \beta_{N \ell, t+1} \stackrel{d}{=} \sum_{\ell=1}^{N} I_{t \ell} I_{t+1, \ell}$ is the (random) number of recaptures from wave $t$ to $t+1$.

The next step is to approximate ${\underset{\sim}{\alpha}}_{N t}^{\widehat{\alpha}}{ }_{1}^{\widehat{\delta}}{ }_{N t}$ and ${ }_{2}^{\widehat{\delta}}{ }_{N t}$ in terms of the independent random variables $\beta_{N \ell t}^{*}$. In this direction we have first that, as $N \rightarrow \infty$,

$$
E\left\|\widehat{\alpha}_{N t}-\widehat{\alpha}_{N t}^{*}\right\|^{2} \rightarrow 0
$$

(by Lemma 1 with $\underline{c}_{N \ell t}=n_{t}^{-\frac{1}{2}}\left(\underline{X}_{N \ell t}-{\underset{\sim}{X}}_{N t}\right)$, using (1.1) and (A1)), and

$$
E\left\|_{1} \widehat{\widehat{\delta}}_{N t}-{ }_{1} \widehat{\widehat{\delta}}_{N t}^{*}\right\|^{2} \rightarrow 0
$$


(by Lemma 2 with $\underset{\sim}{\stackrel{c}{\sim}}{ }_{N t}=g_{N t}^{-\frac{1}{2}}\left(Z_{N \ell t}-\bar{Z}_{N t}\right)$, using (1.1) and (A2)), where $\widehat{\alpha}_{N t}^{*}$ and ${ }_{1}^{*} \widehat{\delta}_{N t}^{*}$ are the same as $\widehat{\alpha}_{N t}^{*}, 1, \widehat{\delta} \underset{\sim}{\sim}$ with $\beta_{N \ell t}$ replaced by $\beta_{N \ell t}^{*}$ :

$$
\begin{aligned}
& \stackrel{\widehat{\alpha}}{\sim}_{N t}^{*}=: \sum_{\ell=1}^{N} n_{t}^{-\frac{1}{2}}\left({\underset{\sim}{N}}_{N \ell t}-\overline{\mathbb{X}}_{N t}\right) \beta_{N \ell t}^{*} \\
& 1 \widehat{\widehat{\delta}}_{N t}^{*}=: \sum_{\ell=1}^{N} g_{N t}^{-\frac{1}{2}}\left(Z_{N \ell t}-\bar{Z}_{N t}\right) \beta_{N \ell t}^{*} \beta_{N \ell, t+1}^{*} \\
& =: \sum_{\ell=1}^{N} g_{N t}^{-\frac{1}{2}}\left(Z_{N \ell t}-\bar{Z}_{N t}\right)\left[\beta_{N \ell t}^{*} \beta_{N \ell, t+1}^{*}-E\left(\beta_{N \ell t}^{*} \beta_{N \ell, t+1}^{*}\right)\right] .
\end{aligned}
$$

To approximate $2 \underset{\sim}{\widehat{\delta}} N t$ (to which Lemma 2 does not apply), we need

Lemma 3. Let ${ }_{2} \hat{\delta}_{N t}^{*}=: g_{N t}^{-\frac{1}{2}} \bar{Z}_{N t} \sum_{\ell=1}^{N}\left[\beta_{N \ell t}^{*} \beta_{N \ell, t+1}^{*}-E\left(\beta_{N \ell t}^{*} \beta_{N \ell, t+1}^{*}\right)\right]$.

Then

$$
E\left\|_{2} \widehat{\widehat{\delta}}_{N t}-2_{2} \widehat{\hat{\delta}}_{N t}^{*}\right\|^{2} \rightarrow 0 \text { as } N \rightarrow \infty \text {. }
$$

Results (2.4), (2.5) and (2.8) imply that, for arbitrary $\underline{\lambda}$, $E\left\{\lambda_{\sim}^{T} \widetilde{W}_{N}-\lambda^{T} \widetilde{W}_{N}^{*}\right\} \rightarrow 0$ as $N \rightarrow \infty$, where

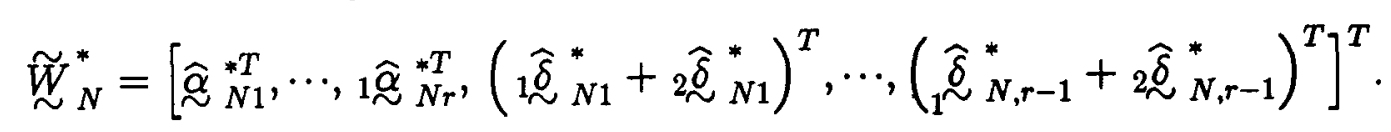

Theorem 1 now follows by the central limit theorem for triangular arrays of row-wise independent variables, ala

Lemma 4. $\lambda_{\sim}^{\mathrm{T}} \widetilde{\mathrm{W}}_{\mathrm{N}}^{*} \stackrel{\mathcal{L}}{\rightarrow} \mathrm{N}(0, \underset{\sim}{0})$ as $\mathrm{N} \rightarrow \infty$.

Proofs of Lemmas 2-4 are given in section 3.

3. Technical Details

Proof of Lemma 2. The $j^{t h}(1 \leq j \leq m)$ element $\epsilon_{N t j}-\epsilon_{N t j}^{*}$ of $\epsilon_{N t}-\epsilon_{N t}^{*}$ has squared expectation (writing $\left.\underset{\sim}{\sim} N k t=\left(c_{N k t}^{(j)}: 1 \leq j \leq m\right)\right)$

$E\left\{\epsilon_{N t j}-\epsilon_{N t j}^{*}\right\}^{2}=E\left\{\sum_{k=1}^{N} c_{N k t}^{(j)}\left(\beta_{N k t} \beta_{N k, t+1}-\beta_{N k t}^{*} \beta_{N k, t+1}^{*}\right)\right\}^{2}$ 


$$
\begin{aligned}
& =E\left\{\sum_{k=1}^{N} c_{N k t}^{(j)}\left(\beta_{N k t} \beta_{N k, t+1}-\beta_{N k t} \beta_{N k, t+1}^{*}+\beta_{N k t} \beta_{N k, t+1}^{*}-\beta_{N k t}^{*} \beta_{N k, t+1}^{*}\right)\right\}^{2} \\
& =E\left\{\sum_{k=1}^{N} c_{N k t}^{(j)} \beta_{N k t}\left(\beta_{N k, t+1}-\beta_{N k, t+1}^{*}\right)\right\}^{2} \\
& +E\left\{\sum_{k=1}^{N} c_{N k t}^{(j)} \beta_{N k, t+1}^{*}\left(\beta_{N k t}-\beta_{N k t}^{*}\right)\right\}^{2} \\
& +E\left[\left\{\sum_{K=1}^{N} c_{N k t}^{(j)} \beta_{N k t}\left(\beta_{N k, t+1}-\beta_{N k, t+1}^{*}\right)\right\}\left\{\sum_{\ell=1}^{N} c_{N \ell t}^{(j)} \beta_{N \ell, t+1}^{*}\left(\beta_{N \ell t}-\beta_{N \ell t}^{*}\right)\right\}\right]
\end{aligned}
$$

But (3.1) $\rightarrow 0$ as $N \rightarrow \infty$, since it equals (by virtue of independence for distinct $t$ )

$$
\begin{gathered}
\sum_{k} \sum_{k^{\prime}} c_{N k t}^{(j)} c_{N k^{\prime} t}^{(j)} E\left(\beta_{N k t} \beta_{N k^{\prime} t}\right) E\left[\left(\beta_{N k, t+1}-\beta_{N k, t+1}^{*}\right)\left(\beta_{N k^{\prime}, t+1}-\beta_{N k^{\prime}, t+1}^{*}\right)\right] \\
=\sum_{k} \sum_{k^{\prime}} c_{N k t}^{(j)} c_{N k^{\prime} t}^{(j)} E\left(I_{t k} I_{t k^{\prime}}\right) E\left[\left(\beta_{N k, t+1}-\beta_{N k, t+1}^{*}\right)\left(\beta_{N k^{\prime}, t+1}-\beta_{N k^{\prime}, t+1}^{*}\right)\right] \\
=\frac{n_{t}\left(n_{t}-1\right)}{N(N-1)} E\left(U_{N t j}-U_{N t j}^{*}\right)^{2} \rightarrow 0
\end{gathered}
$$

by assumption (Al) and Lemma 1, where $\underset{\sim}{\underset{N t}{U}}=\left(U_{N t j}\right)$ and ${\underset{\sim}{N t}}_{N t}^{*}=\left(U_{N t j}^{*}\right)$. The quantity in (3.2) may be similarly handled, and the Cauchy-Schwartz inequality, along with the results for (3.1) and (3.2), handles (3.3). (Q.E.D. Lemma 2)

Proof of Lemma 3 Let $D_{N \ell t}=: \beta_{N \ell t} \beta_{N \ell, t+1}$ and $D_{N \ell t}^{*}=: \beta_{N \ell t}^{*} \beta_{N \ell, t+1}^{*}$. Then the $j^{t h}$ element $\left(1 \leq j \leq m_{z}\right)$ of ${ }_{2} \widehat{\delta}_{\mathrm{Nt}^{t}-2} \widehat{\delta}_{\mathrm{Nt}}^{*}$ has squared expectation

$$
g_{N t}^{-1} \bar{Z}_{N t j}^{2} \sum_{\ell=1}^{N} \sum_{\ell^{\prime}=1}^{N} E\left[\left(D_{N \ell t}-D_{N \ell t}^{*}\right)\left(D_{N \ell^{\prime} t}-D_{N \ell^{\prime} t}^{*}\right)\right] .
$$

The expectation in (3.4), denoted $E_{t t \ell \ell^{\prime}}$, has asymptotic order (see Lemma $3 \mathrm{~A}$ below) 


$$
E_{t t \ell \ell^{\prime}}=\left\{\begin{array}{l}
O\left(n_{(N)}^{\frac{3}{2}} / N^{2}\right), \ell=\ell^{\prime} \\
O\left(n_{(N)}^{3} / N^{4}\right), \ell \neq \ell^{\prime}
\end{array}\right.
$$

Thus the order of (3.4) is, using condition (1.1) on $\left\{Z_{N t k}\right\}$,

$$
\begin{aligned}
& O\left(N / n_{(N)}^{2}\right) \cdot O(1) \cdot\left\{N \cdot O\left(n_{(N)}^{\frac{3}{2}} / N^{2}\right)+N^{2} \cdot O\left(n_{(N)}^{3} / N^{4}\right)\right\} \\
= & O\left(N / n_{(N)}^{2}\right) \cdot O(1) \cdot\left\{O\left(n_{(N)}^{\frac{3}{2}} / N\right)\right\} \\
= & O\left(n_{(N)}^{-\frac{1}{2}}\right) \rightarrow 0 \text { by (A1). (QED) Lemma 3). }
\end{aligned}
$$

Lemma 3A. The asymptotic orders in (3.5) hold.

Proof of Lemma 3A. The proof follows from (3.6) and (3.7) below, by straight-forward calculations using the independence over $t$ of the $\beta_{N \ell t}$ and $\beta_{N \ell t}^{*}$, and the fact that $\left\{\beta_{N \ell t}^{*}: \ell=1, \cdots, N\right\}$ are iid Bernoulli $\left(f_{t}\right)$. (See the technical report by Hawkins et al. (1995b) for details.) The key results are:

$$
\begin{gathered}
E\left(\beta_{N \ell t} \beta_{N \ell t}^{*}\right)=f_{t}+O\left(n_{(N)}^{\frac{1}{2}} / N\right), \\
E\left(\beta_{N \ell t} \beta_{N \ell^{\prime} t}^{*}\right)=g_{t}+O\left(n_{(N)}^{\frac{1}{2}} / N^{2}\right), \ell \neq \ell^{\prime},
\end{gathered}
$$

where $g_{t}=: n_{t}\left(n_{t}-1\right) / N(N-1)$. Since the proofs of these results are involved, we give them here.

To prove (3.6), note that

$$
\begin{aligned}
E\left(\beta_{N \ell t} \beta_{N \ell t}^{*}\right) & =E\left[I\left(R_{N \ell}^{(t)} \leq n_{t}\right) I\left(V_{N \ell}^{(t)} \leq n_{t} / N\right)\right] \\
& =\frac{1}{N} \sum_{k=1}^{N} E\left[I\left(R_{N \ell}^{(t)} \leq n_{t}\right) I\left(V_{N \ell}^{(t)} \leq n_{t} / N\right) \mid R_{N \ell}^{(t)}=k\right] \\
& =\frac{1}{N} \sum_{k=1}^{N} I\left(k \leq n_{t}\right) P\left\{V_{N(k)}^{(t)} \leq n_{t} / N\right\} \\
& =\frac{1}{N} \sum_{k=1}^{n_{t}} \sum_{j=k}^{N}\left(\begin{array}{c}
N \\
j
\end{array}\right) f_{t}^{j}\left(1-f_{t}\right)^{N-j}
\end{aligned}
$$


where the third equality holds since the order statistics $V_{N(l)}^{(t)}$ are independent of the ranks $R_{N l}^{(t)}$, and the fourth equality holds since $V_{N(k)}^{(t)}$ is a uniform order statistic and $f_{t}=n_{t} / N$. Interchanging the order of summation in (3.8) gives

$$
\begin{aligned}
E\left(\beta_{N l t} \beta_{N l t}^{*}\right) & =\frac{1}{N}\left\{\sum_{j=1}^{n_{t}} j\left(\begin{array}{c}
N \\
j
\end{array}\right) f_{t}^{j}\left(1-f_{t}\right)^{N-j}+n_{t} \sum_{j=n_{t+1}}^{N}\left(\begin{array}{c}
N \\
j
\end{array}\right) f_{t}^{j}\left(1-f_{t}\right)^{N-j}\right\} \\
& =\frac{1}{N} \sum_{j=1}^{N} j\left(\begin{array}{c}
N \\
j
\end{array}\right) f_{t}^{j}\left(1-f_{t}\right)^{N-j}+\frac{1}{N} \sum_{j=n_{t}+1}^{N}\left(n_{t}-j\right)\left(\begin{array}{c}
N \\
j
\end{array}\right) f_{t}^{j}\left(1-f_{t}\right)^{N-j} \\
& =f_{t}+R_{N t}, \text { where, with } X_{N} \sim \operatorname{Binomial}\left(N, f_{t}\right) \text { and } Z \sim N(0,1),
\end{aligned}
$$

$$
\begin{aligned}
R_{N t}= & f_{t} P\left\{X_{N}>n_{t}\right\}-\frac{1}{N} E\left\{X_{N} I\left(X_{N}>n_{t}\right)\right\} \\
= & f_{t} P\left\{\frac{X_{N}-N f_{t}}{\sqrt{N f_{t}\left(1-f_{t}\right)}}>0\right\} \\
& -\frac{1}{N} \cdot \sqrt{N f_{t}\left(1-f_{t}\right)}\left[E\left\{\frac{X_{N}-N f_{t}}{\sqrt{N f_{t}\left(1-f_{t}\right)}} I\left(X_{N}>N f_{t}\right)\right\}+\frac{N f_{t} P\left(X_{N}>N f_{t}\right)}{\sqrt{N f_{t}\left(1-f_{t}\right)}}\right] \\
& \approx f_{t} P\{Z>0\}-\sqrt{f_{t}\left(1-f_{t}\right) / N} E(Z I(Z>0))-f_{t} P\{Z>0\} \\
& =O\left(n_{(N)}^{\frac{1}{2}} / N\right) .
\end{aligned}
$$

Thus, (3.6) holds. For (3.7), for $\ell \neq \ell^{\prime}$

$E\left(\beta_{N \ell t} \beta_{N \ell^{\prime} t}^{*}\right)=E\left[I\left(R_{N \ell}^{(t)} \leq n_{t}\right) I\left(V_{N \ell^{\prime}}^{(t)} \leq n_{t} / N\right)\right]$

$$
=\sum_{\mathcal{L}} E\left[I\left(R_{N \ell}^{(t)} \leq n_{t}\right) I\left(V_{N \ell^{\prime}}^{(t)} \leq n_{t} / N\right) \mid \underline{R}_{N}^{(t)}=\tau\right] P\left\{\stackrel{R}{\sim}_{N}^{(t)}=\tau\right\},
$$

(where the sum is over all permutations $\underset{\sim}{\sim}=\left(r_{1}, \cdots, r_{N}\right)$ of the numbers $(1, \cdots, N)$ )

$$
\begin{aligned}
& =\frac{1}{N !} \sum_{\chi} I\left(r_{\ell} \leq n_{t}\right) P\left\{V_{N\left(r_{\ell}\right)}^{(t)} \leq n_{t} / N \mid \underset{\sim}{R} \stackrel{(t)}{N}=\underset{\sim}{\sim}\right\} \\
& =\frac{1}{N !} \sum_{\chi} I\left(r_{\ell} \leq n_{t}\right) P\left\{V_{N\left(r_{\ell^{\prime}}\right)}^{(t)} \leq n_{t} / N\right\} \\
& =\frac{1}{N !} \sum_{\Sigma} \sum_{j=1}^{n_{t}} I\left(r_{\ell}=j\right) P\left\{V_{N\left(r_{\ell^{\prime}}\right)}^{(t)} \leq n_{t} / N\right\}
\end{aligned}
$$




$$
=\frac{1}{N !} \sum_{j=1}^{n_{t}} \sum_{\Gamma A_{\ell_{j}}} P\left\{V_{N\left(r_{\ell^{\prime}}\right)}^{(t)} \leq n_{t} / N\right\}
$$

(where $\left.A_{\ell j}=\left\{\tau=\left(r_{1}, \cdots, r_{N}\right): r_{\ell}=j\right\}\right)$

$$
=\frac{1}{N !} \sum_{j=1}^{n_{t}} \sum_{r_{\ell^{\prime}}=1}^{N} I\left(r_{\ell^{\prime}} \neq j\right)(N-2) ! P\left\{V_{N\left(r_{\ell^{\prime}}\right)}^{(t)} \leq n_{t} / N\right\}
$$

(since the probability in (3.9) depends on $I$ only through $r_{\ell^{\prime}}$ )

$$
\begin{aligned}
& =\frac{1}{N(N-1)} \sum_{j=1}^{n_{t}} \sum_{k=1}^{N} I(k \neq j) P\left\{V_{N(k)}^{(t)} \leq n_{t} / N\right\} \\
& =\frac{1}{N(N-1)} \sum_{j=1}^{n_{t}} \sum_{k=1}^{N} I(k \neq j)\left\{\sum_{m=k}^{N}\left(\begin{array}{l}
N \\
m
\end{array}\right) f_{t}^{m}\left(1-f_{t}\right)^{N-m}\right\} \\
& =\frac{1}{N(N-1)}\left\{\sum_{j=1}^{n_{t}} \sum_{k=1}^{N} \sum_{m=k}^{N}\left(\begin{array}{l}
N \\
m
\end{array}\right) f_{t}^{m}\left(1-f_{t}\right)^{N-m}-\sum_{j=1}^{n_{t}} \sum_{m=j}^{N}\left(\begin{array}{l}
N \\
m
\end{array}\right) f_{t}^{m}\left(1-f_{t}\right)^{N-m}\right\} \\
& =\frac{n_{t}}{N(N-1)} \sum_{k=1}^{N} \sum_{m=k}^{N}\left(\begin{array}{l}
N \\
m
\end{array}\right) f_{t}^{m}\left(1-f_{t}\right)^{N-m}-\frac{1}{N-1} E\left(\beta_{N \ell t} \beta_{N \ell t}^{*}\right)
\end{aligned}
$$

(compare (3.8)).

But by reversing the sums, the first term in (3.10) equals

$$
\frac{n_{t}}{N(N-1)} \sum_{m=1}^{N} m\left(\begin{array}{c}
N \\
m
\end{array}\right) f_{t}^{m}\left(1-f_{t}\right)^{N-m}=\frac{n_{t}}{N(N-1)} \cdot N f_{t}=\frac{n_{t}}{N-1} f_{t}
$$

Combining this with (3.10) gives

$$
\begin{aligned}
E\left(\beta_{N \ell t} \beta_{N \ell^{\prime} t}^{*}\right) & =\frac{n_{t}}{N-1} f_{t}-\frac{1}{N-1}\left\{f_{t}+O\left(n_{(N)}^{\frac{1}{2}} / N\right)\right\} \\
& =\left(\frac{n_{t}-1}{N-1}\right) f_{t}+O\left(n_{(N)}^{\frac{1}{2}} / N^{2}\right),
\end{aligned}
$$

giving (3.7). (QED Lemma 3A) 


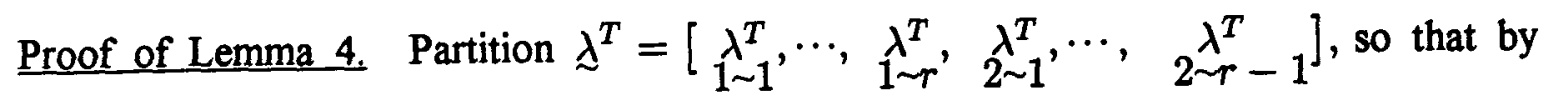
(2.6) and (2.7),

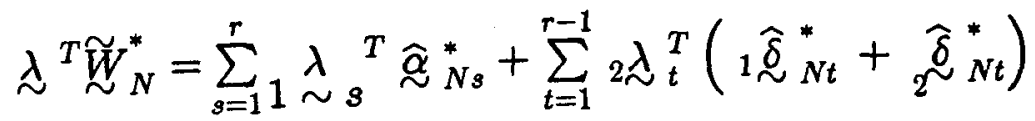

$$
\begin{aligned}
& =\sum_{s=1}^{r} 1_{\sim}^{\lambda}{ }_{s}^{T}\left\{\sum_{\ell=1}^{N}{ }_{1} \nu_{N \ell s} \beta_{N \ell s}^{*}\right\}\left(\text { where }_{1} \nu_{N \ell s}^{\nu}=: n_{s}^{-\frac{1}{2}}\left({\underset{\sim}{N}}_{N \ell s}-\bar{\sim}_{N s}\right)\right) \\
& +\sum_{t=1}^{r-1} \underset{2}{\sim}{ }_{t}^{T}\left\{\sum_{\ell=1}^{N} 2{ }_{\nu}^{\nu}{ }_{N \ell t}\left[\beta_{N \ell t}^{*} \beta_{N \ell, t+1}^{*}-E\left(\beta_{N \ell t}^{*} \beta_{N \ell, t+1}^{*}\right)\right]\right\}
\end{aligned}
$$

$\left(\right.$ where $\left.{ }_{2}^{\mathcal{L}}{ }_{N \ell t}=: g_{N t}^{-\frac{1}{2}} Z_{N \ell t}\right)$

$$
\begin{aligned}
& =\sum_{\ell=1}^{N} \sum_{s=1}^{r}\left\{{ }_{1}{ }_{1}{ }_{s}^{T}{ }_{1}{ }_{N \ell s}\right\} \beta_{N \ell s}^{*} \\
& +\sum_{\ell=1}^{N} \sum_{t=1}^{r-1}\left\{{ }_{2}{ }_{2}^{T}{ }_{t}^{T}{ }_{2}{ }_{N \ell t}\right\}\left[\beta_{N \ell t}^{*} \beta_{N \ell, t+1}^{*}-E\left(\beta_{N \ell t}^{*} \beta_{N \ell, t+1}^{*}\right)\right] \\
& =: \sum_{\ell=1}^{N} \sum_{s=1}^{r}{ }_{1} \gamma_{N \ell s} \beta_{N \ell s}^{*}+\sum_{\ell=1}^{N} \sum_{t=1}^{r-1}{ }_{2} \gamma_{N \ell t}\left[\beta_{N \ell t}^{*} \beta_{N \ell, t+1}^{*}-E\left(\beta_{N \ell t}^{*} \beta_{N \ell \ell+1}^{*}\right)\right] \\
& =\sum_{\ell=1}^{N} \eta_{N \ell}, \text { say, where }
\end{aligned}
$$

$\eta_{N \ell}=: \sum_{s=1}^{r}{ }_{1} \gamma_{N \ell s} \beta_{N \ell s}^{*}+\sum_{t=1}^{r-1}{ }_{2} \gamma_{N \ell t}\left[\beta_{N \ell t}^{*} \beta_{N \ell, t+1}^{*}-E\left(\beta_{N \ell t}^{*} \beta_{N \ell, t+1}^{*}\right)\right]$

Now the $\left\{\eta_{\mathrm{N} \ell}: 1 \leq \ell \leq N, N \geq 1\right\}$ form a triangular array of row-wise independent random variables, so it is sufficient for $\sum_{\ell=1}^{N} \eta_{N \ell}$ to be asymptotically $N\left(\bar{A}_{N}, \bar{B}_{N}^{2}\right)$ (i.e. $B_{N}^{-1}\left(\sum \eta_{N \ell}-\bar{A}_{N}\right) \stackrel{\mathcal{L}}{\rightarrow} N(0,1)$; see Serfling (1980), p.31) to verify the Lindeberg condition : 


$$
\forall \epsilon>0, \bar{B}_{N}^{-2} \sum_{\ell=1}^{N} E\left\{\left(\eta_{N \ell}-E \eta_{N \ell}\right)^{2} I\left(\left|\eta_{N \ell}-E \eta_{N \ell}\right|>\epsilon \bar{B}_{N}\right)\right\} \rightarrow 0
$$

as $N \rightarrow \infty$, where $\bar{A}=: \sum_{\ell=1}^{N} E \eta_{N \ell}$ and $\bar{B}_{N}^{2}=: \sum_{\ell=1}^{N} \operatorname{Var}\left(\eta_{N \ell}\right)$. Since the quantity in (3.12) is (by Cauchy-Schwartz) dominated by

$$
\left.\bar{B}_{N}^{-2} \sum_{\ell=1}^{N}\left\{E\left(\eta_{N \ell}-E \eta_{N \ell}\right)^{4}\right\}^{\frac{1}{2}} P^{\frac{1}{2}}\left\{\left|\eta_{N \ell}-E \eta_{N \ell}\right|>\epsilon \bar{B}_{N}\right)\right\}
$$

we shall show that (3.13) $\rightarrow 0$.

First we obtain the order of $\bar{B}_{N}^{2}$. Using the fact that $\beta_{N \ell t}^{*}$ has the Bernoulli $\left(p=f_{t}\right.$ ) distribution, iid over $\ell=1, \cdots, N$ for fixed $t$, and independent for distinct $t$, we compute as follows:

$$
\begin{aligned}
\operatorname{Var}\left(\eta_{N \ell}\right) & =\operatorname{Var}\left(\sum_{s}{ }_{1} \gamma_{N \ell s} \beta_{N \ell s}^{*}\right) \\
& +\operatorname{Var}\left(\sum_{t}{ }_{2} \gamma_{N \ell t} \beta_{N \ell t}^{*} \beta_{N \ell, t+1}^{*}\right) \\
& +2 \operatorname{cov}\left(\sum_{S}{ }_{1} \gamma_{N \ell s} \beta_{N \ell s}^{*}, \sum_{t}{ }_{2} \gamma_{N \ell t} \beta_{N \ell t}^{*} \beta_{N \ell, t+1}^{*}\right)
\end{aligned}
$$

Then we have

$$
\begin{aligned}
(3.14) & =\sum_{s} \gamma_{N \ell s}^{2} \operatorname{Var}\left(\beta_{N \ell s}^{*}\right)=\sum_{s}{ }_{1} \gamma_{N \ell s}^{2} f_{s}\left(1-f_{s}\right) \\
& +\sum_{t} \sum_{t} \gamma_{N \ell t}^{2} \operatorname{Var}\left(\beta_{N \ell t}^{*} \beta_{N \ell, t+1}\right) \\
& =\sum_{t} \gamma_{N \ell t_{2}} \gamma_{N \ell t^{\prime}} \operatorname{cov}\left(\beta_{N \ell t}^{2}{ }_{N \ell t} \beta_{N \ell, t+1} f_{t+1}\left(1-\beta_{N \ell t^{\prime}}^{*} \beta_{N \ell, t^{\prime}+1}^{*}\right)\right. \\
& +\sum_{t=1}^{r-1}\left\{{ }_{2} \gamma_{N \ell t}\right)
\end{aligned}
$$

and 
(3.16) $=2 \sum_{t=1}^{r-1}\left\{{ }_{2} \gamma_{N \ell t}{ }_{2} \gamma_{N \ell t} f_{t} f_{t+1}\left(1-f_{t}\right)+{ }_{1} \gamma_{N \ell, t+1}{ }_{2} \gamma_{N \ell t} f_{t} f_{t+1}\left(1-f_{t+1}\right)\right\}$

From (3.17)-(3.19) it follows by routine calculations that, as $N \rightarrow \infty$,

$$
\begin{aligned}
& \bar{B}_{N}^{2}=\sum_{\ell=1}^{N} \operatorname{Var}\left(\eta_{N \ell}\right) \rightarrow \\
& \sum_{s=1}^{r}{ }_{1} \lambda_{s}{ }_{s}^{T} \Gamma_{s}^{x} 1_{\sim}^{\lambda}
\end{aligned}
$$

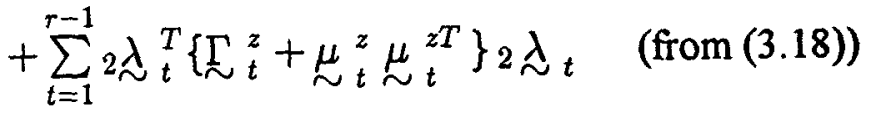

$$
\begin{aligned}
& +0 \quad \text { (from (3.19)). }
\end{aligned}
$$

(The second term in (3.18) is $O\left(n_{(N)} / N\right)$, and (3.19) is $O\left(\left(n_{(N)} / N\right)^{\frac{1}{2}}\right)$.) Thus,

$$
\bar{B}_{N}^{2} \text { is } O(1) \text { as } N \rightarrow \infty \text {. }
$$

Next we obtain the order of the numerator in (3.13). First, one checks that the fourth central moment of the Bernoulli $(p)$ distribution is $p(1-p)\left[p^{3}+(1-p)^{3}\right]$. Then by repeated application of the $C_{r}$-inequality (see Rao (1973), p. 149) there are finite positive constants $C_{1}, C_{2}, C_{3}$ such that

$$
\begin{aligned}
E\left(\eta_{N \ell}-E \eta_{N \ell}\right)^{4} & =E\left\{\sum_{s=1}^{r}{ }_{1} \gamma_{N \ell s}\left(\beta_{N \ell s}^{*}-\mathrm{E} \beta_{N \ell s}^{*}\right)\right. \\
& +\sum_{t=1}^{r-1}{ }_{2} \gamma_{N \ell t}\left[\beta_{N \ell t}^{*} \beta_{N \ell, t+1}^{*}-E\left(\beta_{N \ell t}^{*} \beta_{N \ell, t+1}^{*}\right)\right\}^{4} \\
& \leq C_{1}\left\{E\left[\sum_{s=1}^{r}{ }_{1} \gamma_{N \ell s}\left(\beta_{N \ell s}^{*}-E \beta_{N \ell s}^{*}\right)\right]^{4}\right. \\
& \left.+E\left[\sum_{t=1}^{r-1}{ }_{2} \gamma_{N \ell t}\left(\beta_{N \ell t}^{*} \beta_{N \ell, t+1}^{*}-E\left(\beta_{N \ell t}^{*} \beta_{N \ell, t+1}^{*}\right)\right)\right]^{4}\right\} \\
& \leq C_{1}\left\{C_{2} \sum_{s=1}^{r}{ }_{1} \gamma_{N \ell s}^{4} E\left(\beta_{N \ell s}^{*}-E \beta_{N \ell s}^{*}\right)^{4}\right.
\end{aligned}
$$




$$
\left.+C_{3} \sum_{t=1}^{r-1}{ }_{2} \gamma_{N \ell t}^{4} E\left[\beta_{N \ell t}^{*} \beta_{N \ell, t+1}^{*}-E\left(\beta_{N \ell t}^{*} \beta_{N \ell, t+1}^{*}\right)\right]^{4}\right\}
$$

Finally, since $\beta_{N \ell t}^{*} \sim$ Bernoulli $\left(p=f_{t}\right)$ and $\beta_{N \ell t}^{*} \beta_{N \ell, t+1}^{*} \sim$ Bernoulli $\quad\left(p=f_{t} f_{t+1}\right)$, we have (writing $n_{(N)} \equiv n$ for the moment)

$$
\begin{gathered}
E\left(\beta_{N \ell s}^{*}-E \beta_{N \ell s}^{*}\right)^{4}=O\left(f_{s}\right)=O(n / N), \\
E\left[\beta_{N \ell t}^{*} \beta_{N \ell, t+1}^{*}-E\left(\beta_{N \ell t}^{*} \beta_{N \ell, t+1}^{*}\right)\right]^{4}=O\left(f_{t} f_{t+1}\right)=O\left(\left(\frac{n}{N}\right)^{2}\right) .
\end{gathered}
$$

Combining (3.22)-(3.24) gives

$$
\begin{aligned}
E\left(\eta_{N \ell}-E \eta_{N \ell}\right)^{4} & =O\left[\left(\frac{n}{N}\right) \sum_{s}{ }_{1} \gamma_{N \ell s}^{4}\right]+O\left[\left(\frac{n}{N}\right)^{2} \sum_{t}{ }_{2} \gamma_{N \ell t}^{4}\right] \\
& =O\left[\left(\frac{n}{N}\right) \frac{1}{n^{2}}\right]+O\left[\left(\frac{n}{N}\right)^{2} g_{N}^{-2}\right] \\
& =O\left(\frac{1}{n N}\right)+O\left(\frac{1}{n^{2}}\right)=O\left(\frac{1}{n^{2}}\right) .
\end{aligned}
$$

Also, by the Chebychev-type inequality and the preceding analysis,

$$
P\left\{\left|\eta_{N \ell}-E \eta_{N \ell}\right|>\epsilon \bar{B}_{N}\right\} \leq \epsilon^{-4} \bar{B}_{N}^{-4} E\left(\eta_{N \ell}-E \eta_{N \ell}\right)^{4}=O\left(\frac{1}{n^{2}}\right)
$$

In view of assumption (1.1), the sum in (3.13) is therefore of order

$$
N\left[O\left(\frac{1}{n^{2}}\right)\right]^{\frac{1}{2}}\left[O\left(\frac{1}{n^{2}}\right)\right]^{\frac{1}{2}}=O\left(N / n^{2}\right) .
$$

By (3.21) and assumption $(A 2),(3.13) \rightarrow 0$, giving the Lindeberg condition (3.12). To see that the asymptotic mean of $\underset{\sim}{T} \widetilde{W}_{N}^{*}$ is zero, we simply check that $\bar{A}_{N}=0$, which follows easily from (3.11). The form of $\sum_{\sim}^{N}$ follows from (3.20).

(Q.E.D. Lemma 4)

\section{REFERENCES:}

Alho, J. M., Mulry, M. H., Wurdeman, K. and Kim, J. (1993). Estimating heterogeneity in the probabilities of enumeration for dual-system estimation. Journal of the American Statistical Association, 88, 1130-1136.

Chandrasekaran, C. and Deming, W. E. (1949) On a method of estimating birth and death rates and the extent of registration. Journal of the American Statistical 
Association, 44, 101-115.

Hajek, J. (1960). Limiting distributions in simple random sampling from a finite population. Publications of the Mathematics Institute of the Hungarian Academy of Science 5, 361-374.

Hawkins, D. L., Han, C. P. and Eisenfeld, J. (1995a). Estimating transition probabilities from aggregate samples augmented by haphazard recaptures. To appear in Biometrics.

Hawkins, D. L., Han, C. P. and Eisenfeld, J. (1995b). Estimating transition probabilities from aggregate samples augmented by haphazard matches. Technical report \#302. Department of Mathematics, University of TexasArlington, Arlington, TX 76019.

Hawkins, D.L. and Han, C.P. (1996). A central limit theorem for certain nonlinear statistics in repeated sampling of a finite population. Technical Report \#312, Department of Mathematics, University of Texas at Arlington, Arlington, Texas 76019.

Isaki, Cary T. (1986). Bias of the dual system estimator and some alternatives. Communications in Statistics-Theory and Methods, 15, 1435-1450.

Isaki, Cary T. and Schultz, L. K. (1987). The effects of correlation and matching error on dual system estimation. Communications in Statistics-Theory and Methods, $16,2405-2427$.

Krotki, K. J. (1978). Developments in Dual System Estimation of Population Size and Growth. The University of Alberta Press: Edmonton, Alberta.

Madow, W. G. (1948). On the limiting distributions of estimates based on samples from finite universes. Annals of Mathematical Statistics 19, 535-545.

Rao, C. R. (1976). Linear Statistical Inference. Wiley.

Rosen, B. (1965). Limit theorems for sampling from finite populations. Arkiv for Matematik 5, 383-424.

Serfling, R. J. (1980). Approximation Theorems of Mathematical Statistics, Wiley.

White, D. B. (1988). An application of a multivariate central limit theorem to sampling without replacement. Journal of Multivariate Analysis 24, 123-128. 